\title{
COLLINEATION GROUPS OF TRANSLATION PLANES OF SMALL DIMENSION
}

\author{
T.G. OSTROM \\ Department of Pure and Applied Mathematics \\ Washington State University \\ Pullman, Washington 99164 U.S.A. \\ (Received September 3, 1980 and in revised form March 30, 1981)
}

ABSTRACT. A subgroup of the linear translation complement of a translation plane is geometrically irreducible if it has no invariant lines or subplanes. A similar definition can be given for "geometrically primitive". If a group is geometrically primitive and solvable then it is fixed point free or metacyclic or has a normal subgroup of order $w^{2 a+b}$ where $w^{a}$ divides the dimension of the vector space. Similar conditions hold for solvable normal subgroups of geometrically primitive nonsolvable groups. When the dimension of the vector space is small there are restrictions on the group which might possibly be in the translation complement. We look at the situation for certain orders of the plane.

KEY WORDS AND PHRASES. Translation planes, translation complement, linear groups. 1980 MATHEMATICS SUBJECT CLASSIFICATION CODES. 51A40, $20 \mathrm{H} 30$.

\section{INTRODUCTION.}

A translation plane $\pi$ of order $q^{d}$ with kernel $G F(q)=F$ can be represented as follows: Let $\mathrm{V}$ be a vector space of dimension $2 \mathrm{~d}$ over $\mathrm{F}$. A spread defined on $\mathrm{V}$ is a class of d-dimensional subspaces (called the components of the spread) such that each nonzero element of $\mathrm{V}$ belongs to exactly one component. The points of $\pi$ are the elements of $\mathrm{V}$, the lines of $\pi$ are the components of the spread and their translates. The group of nonsingular semi-linear transformations of $\mathrm{V}$ which permute the components is called the translation complement of $\pi$. The subgroup consisting of linear transform- 
ations is the linear translation complement.

We are interested in finding information as to which abstract groups can act as subgroups of the linear translation complement; what the nature of the action is and also what the nature of the plane is. The action is fully as important as the (abstract) group.

A particularly simple kind of action is for the group to be fixed-point-free (f.p.f.). A linear group is fixed point free if no nontrivial element fixes any nonzero vector. The translations and a fixed-point-free group generate a Frobenius group with the f.p.f. group as Frobenius complement. A normal subgroup $G_{1}$ of a non-f.p.f. group is a minimal non-f.p.f. group with respect to $G$ if it is non-f.p.f. but every normal subgroup of $G$ properly contained in $G_{1}$ is $f . p . f$. It can happen that a minimal non-f.p.f. group with respect to $G$ is also a minimal normal nonsolvable subgroup. This situation has been analyzed in previous papers and some of the results are given in (2.4) below.

In (2.6) we show that if $G$ is solvable then, subject to certain irreducibility requirements at least one of the following holds: (1) G is f.p.f. (2) G is metacyclic (3) $G$ has a normal subgroup $W$ which is a $w$-group of order $w^{2 a+b}$ for some a and $b$ and $w^{a}$ divides the dimension $2 d$ of the vector space. The nature of $G$ and 1 ts action is much easier to analyze when case (3) does not occur. A similar situation occurs in the nonsolvable case. See (2.3) and (2.4).

In (2.7), (2.8) and (2.9) we develop some circumstances under which case (3) cannot occur. In Section 3 we develop some lower bounds on the value of $d$ if the plane is to admit $\operatorname{SL}(2, u)$ or $\operatorname{PSL}(2, u)$ for a given u relatively prime to $q$. (Recall that the plane is defined on a vector space of dimension $2 d$ over GF(q)). This is a slight sharpening of some standard results. (See Harris and Hering [2].) However this sharper result is useful in looking at particular cases.

We confess to a poor background in group representation theory. All of this is representation theory in some sense and may be implied by results in classical representation theory. We would be pleased if some expert could show us how to get our results from standard representation theory if this could be done in substantially less space than we have used to get them directly by fairly elementary means. 
What we seem to be ending up with are results which show that when d and the characteristic of $\mathrm{GF}(\mathrm{q})$ are suitably restricted, the number of groups to be considered is reasonably small.

In Section 4 we illustrate how this works for some specific values of $d$ with $q$ odd or even.

DEFINITION 1.1. If $\mathrm{G}$ is a group of nonsingular transformations, $V(G)$ denotes the vector subspace consisting of all vectors fixed by $G$. If $\sigma$ is an element of $G$, $\mathrm{V}(\sigma)$ means the same as $\mathrm{V}(\langle\sigma\rangle)$

DEFINITION 1.2. The prime $u$ is a $q$-primitive divisor of $q^{d}-1$ if $u$ divides $q^{d}-1$ but $u$ does not divide $q^{a}-1$ for $0<a<d$.

NOTATION 1.3. If $\mathrm{G}$ is a group, $\mathrm{Z}(\mathrm{G})$ denotes the center of $\mathrm{G}$. Whenever we are considering subgroups of a given group G, C(H) will denote the centralizer of $\mathrm{H}$ in $G$. If $G_{0}$ is another subgroup of $G$, the centralizer of $H$ in $G_{0}$ will be denoted by $G_{0} \cap c(H)$.

We shall make repeated use of the fact that the Sylow subgroups of a Frobenius complement (and hence of an f.p.f. group) are cyclic or generalized quaternion.

This research was supported by the National Science Foundation.

2. SOLVABLE NON-F.P.F. GROUPS.

DEFINITION 2.1. Let $G$ be a subgroup of the linear translation complement of a translation plane $\pi$. Then $G$ will be said to be geometrically irreducible either if $G$ is irreducible as a group of linear transformations or if none of the invariant vector subspaces is a proper subplane of $\pi$ or is a component of the spread defining $\pi$. If $G$ is geometrically irreducible, $G$ will be said to be geometrically primitive if $\pi$ (as a vector space) cannot be written as a direct sum of proper subspaces which are subplanes or components of the spread and are permuted by $\mathbf{G}$.

REMARK. Recall that if $\sigma$ is a nontrivial member of the linear translation complement then the subspace $V(\sigma)$ pointwise fixed by $\sigma$ is either a proper subplane or is a (not necessarily proper) subspace of a component. A subgroup $G$ of the linear translation complement is not geometrically irreducible if $\mathrm{G}$ has a normal 
subgroup $H \neq 1$ such that $\mathrm{V}(\mathrm{H})$ is nontrivial.

LEMMA 2.2. If $G$ is geometrically primitive then $G$ every normal elementary abelian subgroup is cyclic of prime order.

PROOF. Suppose that G $\triangleright W$ where $W$ is an elementary abelian w-group of order $w^{a}$, where $a>1$ and $w$ is prime. Then $w$ cannot be fixed point free and for some element $\sigma \in \mathrm{W}, \mathrm{V}(\sigma)$ is nontrivial. Furthermore $\mathrm{V}(\sigma)$ is invariant under W.

Let $\mathrm{V}$ be a minimal invariant G-space. By Clifford's Theorem $\mathrm{V}=\mathrm{v}_{1} \oplus \ldots \oplus \mathrm{v}_{\mathrm{k}}$ where $v_{1}, v_{2}$, etc. are homogeneous $\mathrm{W}$-spaces--i.e. the minimal $\mathrm{W}$-spaces in $\mathrm{v}_{1}$ are isomorphic as $W$-modules. If an element $\sigma$ of $W$ is non-f.p.f. on $v_{i}$, it fixes a minimal $\mathrm{W}$-space pointwise. Hence every minimal $\mathrm{W}$-space in $\mathrm{V}_{i}$ is pointwise fixed by $\sigma$ so $v_{1}$ itself is pointwise fixed by $\sigma$. Clifford's Theorem also says that the $V_{i}$ are subspaces of imprimitivity for $G$.

Let $\mathrm{w}_{1}$ be the subgroup of $\mathrm{w}$ which fixes $v_{1}$ pointwise. Let $v_{1}^{*}, v_{2}^{*}, \ldots, v_{k}^{*}$ be distinct images of $\mathrm{V}\left(\mathrm{W}_{1}\right)$ under $G$. Note that $\mathrm{V}\left(\mathrm{W}_{1}\right)$ need not be a subspace of $\mathrm{V}$. Suppose that $v_{1}^{*}+v_{2}^{*}+\ldots+v_{k-1}^{*}=v_{1}^{*} \oplus v_{2}^{*} \ldots \ldots \oplus v_{k-1}^{*}=\hat{v}$ and that $\hat{v} \cap v_{k}^{*}$ is nontrivial. Note that $v_{1}^{*}, v_{2}^{*}$, etc. are invariant under $w$ and each is pointwise fixed by some conjugate of $w_{1}$. It follows that $v_{k}^{*} \cap v_{i}^{*}$ is nontrivial for some $1=1,2, \ldots, \mathrm{h}-1$. Thus there are two conjugates, say $\lambda_{1}^{-1} \mathrm{~W}_{1} \lambda_{1}$ and $\lambda_{2}^{-1} \mathrm{~W}_{1} \lambda_{2}$ both fixing $v_{k}^{*} \cap v_{i}^{*}$ pointwise. Hence there is some homogeneous $w$-space $v_{j}$ which is pointwise fixed by $\lambda_{1}^{-1} \mathrm{w}_{1} \lambda_{1}$ and $\lambda_{2}^{-1} \mathrm{w}_{1} \lambda_{2}$. But the subgroup of $w$ which fixes $v_{j}$ pointwise is some conjugate of $\mathrm{w}_{1}$. Hence $\lambda_{1}^{-1} \mathrm{w}_{1} \lambda_{1}=\lambda_{2}^{-1} \mathrm{w}_{1} \lambda_{2}$ so that $\mathrm{v}_{\mathrm{k}}^{*}=\mathrm{v}_{i}^{*}$. It follows that $\pi$ is a direct sum of $V\left(W_{1}\right)$ and its distinct images under $G$ contrary to the condition that $G$ is geometrically primitive.

THEOREM 2.3. Let $G$ be a nonsolvable subgroup of the linear translation complement of a finite translation plane $\pi$. Let $G_{0}$ be a minimal nonsolvable normal subgroup of $G$ and let $H$ be a maximal normal subgroup of $G$ included in $G_{0}$ but not equal to $G_{0}$. Then either $H$ is fixed point free or $G_{0}$ contains a subgroup $W$ which is minimal nonfixed point free with respect to $G$. Furthermore $W$ is a w-group for some prime $\mathrm{w}$ and $\mathrm{W} / \mathrm{W}_{0}$ is elementary abelian, where $\mathrm{w}_{0}$ is the maximal normal subgroup of $G$ included in $W$ but not equal to $W$. 
THEOREM 2.4. In $(2.3)$ if $H$ is fixed point free then $H=Z\left(G_{0}\right)$. If $H$ is not fixed point free so that $W$ exists, then either $W$ is elementary abelian or $\left|w / w_{0}\right|=w^{2 a}$ where $w^{a}$ divides the dimension of the vector space on which $\pi$ is defined and the group of automorphisms of $W / W_{0}$ induced by conjugation with respect to $G$ is isomorphic to a subgroup of $\mathrm{Sp}(2 \mathrm{a}, \mathrm{w})$.

The above two theorems are contained in Lemma (2.2) and (2.8) of our paper on planes of odd order and dimension [9] and Lemma (2.5) of our paper on planes of even order in which the dimension has one odd factor [10]. The key to (2.4) above is Huppert's Satz 13.7 Chapter III in his book [5].

We now return to the solvable case

LEMMA 2.5. Let $G$ be a solvable group of linear transformations acting on a vector space $V$ of dimension $2 d$ over GF( $q)$. Then at least one of the following holds: (1) G is fixed point free; (2) G is metacyclic; (3) G has a normal subgroup $W$ with the properties of $W$ in (2.3) and $W_{0}$ is cyclic; (4) $G$ has a normal subgroup $Q$ isomorphic to the quaternion group of order 8 and if $G_{1}$ is a minimal non-f.p.f. group with respect to $G$ then either the non-f.p.f. elements in $G_{1}$ have order 2 or 3 or $G_{1}$ centralizes $Q$.

PROOF. Suppose that $G$ is not fixed point free. Let 2 be a maximal normal subgroup of $G$. If $\mathcal{U}$ is not cyclic then $\mathcal{U}$ is not $f . p . f$. and $\mathcal{U}$ contains a subgroup W which is minimal non-f.p.f. group with respect to G.

By Corollary (3.3) of [8] if $W$ is a solvable minimal non-f.p.f. group with respect to $G$ and $W_{0}$ is the maximal normal subgroup $\neq W$ of $G$ included in $G$, then $\mathrm{W} / \mathrm{W}_{0}$ is elementary abelian.

Hence we have conclusion (3) if $\mathscr{U}$ is not cyclic. Suppose that $\mathcal{A}$ is cyclic.

If $\mathcal{U}$ is its own centralizer in $G$, then $G / \mathscr{U}$ is cyclic. In this case we have conclusion (2) since the outer automorphism group of a cyclic group is cyclic.

If $\mathscr{U}$ is not its own centralizer in $G$, then the centralizer of $\mathcal{Q}$ in $G$ contains a minimal normal nonabelian subgroup $W^{*}$ of $G$. The conditions of Huppert's Hilfsatz I are satisfied [4] and the conclusions include the following:

1. $\mathrm{W}^{*}$ has prime power order, say $\mathrm{w}^{\mathrm{a}}$.

2. $W^{*} \bmod i t s$ center is elementary abelian. 
3. If $w>2$ and $\sigma \in W$ then $\sigma^{w}=1$.

4. If $w=2$ and $\sigma \in W$ then $\sigma^{4}=1$.

Thus if $w \neq 2$, we may take $W^{\star}=W$ and we have our conclusion (3). We also have conclusion (3) if $w=2$ and $W^{*}$ is not f.p.f. Otherwise $w=2$, $W^{*}$ is f.p.f. so $W^{*}$ is generalized quaternion. But if $W^{*}$ has exponent 4 this implies that $W^{*}$ is quaternion of order 8. Let us change our notation and let $Q$ be this normal quaternion group.

Suppose that $G$ has a minimal non-f.p.f. group $G_{1}$ which does not centralize $Q$. Since $G_{1}$ must be generated by its non-f.p.f. elements, it follows that $G_{1}$ contains an element which induces a nontrivial automorphism on $Q$ by conjugation. But the outer automorphism group of $\mathrm{Q}$ is isomorphic to $\mathrm{S}_{4}$ so its order must divide 24 . This gives us case (4) of the Lemma.

THEOREM 2.6. Let $\pi$ be a translation plane of order $q^{d}$ and kernel GF (q). Let G be a solvable subgroup of the linear translation complement which is geometrically irreducible and geometrically primitive. Then at least one of the following holds: (1) G is fixed point free. (2) G is metacyclic. (3) G has a normal subgroup $W$ such that $W$ is a $w-g r o u p$ for some prime $w$. $W \bmod i t s$ center $W_{0}$ is elementary abelian of order $w^{2 a}$ for some $a>1$ where $w^{a}$ divides $2 d$. The group induced by $G$ on $\mathrm{W} / \mathrm{W}_{0}$ by conjugation is isomorphic to a subgroup of $\operatorname{SP}(2 \mathrm{a}, \mathrm{w})$.

PROOF. Apply (2.2), (2.5) and Hilfsatz II from Huppert's paper [4] or Satz 13.7 Chapter III in his book [5]. Note that both cases (3) and (4) of (2.5) come under case (3) of the present theorem.

The situation at this stage can briefly be described by saying that if $G$ is geometrically irreducible and geometrically primitive then every solvable normal subgroup is fixed point free and if $G$ has a minimal nonsolvable normal subgroup $G_{0}$ then $G_{0}$ modulo its center is a direct product of isomorphic simple groups.

Note that $G_{0}$ is its own derived group and (see Huppert [5] Hilfsatz 5.23.3) in this case $Z\left(G_{0}\right)$ is a subgroup of the Schur multiplier of $G_{0} / Z\left(G_{0}\right)$. The situation where $G_{0}$ contains a (noncyclic) metacyclic normal subgroup of $G$ does not arise when $G$ is nonsolvable due to the fact that $G_{0}$ is a minimal normal subgroup of $\mathrm{G}$.

We can now drop the irreducibility considerations and consider the cases where 
$\mathrm{G}_{0} / \mathrm{Z}\left(\mathrm{G}_{0}\right)$ is simple. As we shall see the possibility frequently arises in translation $p$ lanes that $G_{0} / Z\left(G_{0}\right)$ is $\operatorname{PSL}(2, u)$ for some $u$. For $u \neq 9$, the order of the Schur multiplier divides 2 so that $G_{0}$ turns out to be $\operatorname{SL}(2, u)$ or $\operatorname{PSL}(2, u)$ in these situations.

We might remark that we have not made much use of the geometry and that primitivity as a linear group is at least as strong a condition as geometrical primitivity.

In any case it is desirable to have conditions in which every solvable normal subgroup is fixed point free.

We find it convenient to use the expression "geometrically primitive" instead of the more complete "geometrically irreducible and geometrically primitive".

COROLLARY 2.7. Let $\pi$ be a translation plane of order $q^{d}$ with kernel GF(q). Suppose that both $q$ and $d$ are powers of the same prime $u$. Let $G$ be a solvable geometrically primitive subgroup of the linear translation complement. Then no minimal non-f.p.f. group with respect to $G$ is a w-group for a prime w.

PROOF. At characteristic u a u-group has a nontrivial subspace which it fixes pointwise. If $W$ exists with $W / Z(W)$ of order $w^{2 a}$ where $w^{a}$ divides $2 d$ it follows in this case that $w=u$. Hence $W$ cannot exist. The possibility that $W$ might be elementary abelian is excluded by the geometrical primitivity.

If $q^{d}-1$ has a $q$-primitive divisor $u$ (see Definition (1.2)) and if the stabilizer of some component of the spread is transitive on nonzero points, then the order of the translation complement is divisible by $u$. Kallaher and the author made much use of this idea [6].

LEMMA 2.8. Let $G$ be a geometrically primitive subgroup of the linear translation complement of a translation plane of order $q^{d}$ with kernel GF(q). Let $d^{*}$ be the largest prime power factor of $2 d$ and let $\hat{d}$ be the largest prime power factor of $d$. Suppose that $u$ is a prime factor of $|G|$ such that $u>\hat{d}+1$ if $q$ is even or $u \geqslant \frac{1}{2}\left(d^{\star}+1\right)$ and $u \neq d^{\star}$ if $q$ is odd. Suppose that $G$ has a normal subgroup $W$ as in (2.3) and (2.4) or (2.5). Then all elements of order $u$ in G centralize W.

PROOF. The order of $\operatorname{Sp}(2 a, w)$ is 


$$
\left(w^{2 a}-1\right)\left(w^{2 a-2}-1\right) \cdots\left(w^{2}-1\right) w^{a^{2}} .
$$

Under the hypotheses if $\left|\mathrm{w} / \mathrm{w}_{0}\right|=w^{2 a}$, where $w^{a}$ divides $2 d$, the prime $u$ does not divide $|S p(2 a, w)|$. Thus if $w$ exists and $\sigma$ is some element of order $u$ in $G$ and $\lambda \in W$ we must have that $\sigma^{-1} \lambda \sigma=\lambda \nu$ for some $\nu$ in $W_{0}$ since $\sigma$ must induce the trivial automorphism of $\mathrm{w} / \mathrm{w}_{0}$. Then $\lambda=\sigma^{-\mathrm{u}} \lambda \sigma^{\mathrm{u}}=\lambda \nu^{\mathrm{u}}$ so that $\nu=1$ since $(\mathrm{u}, \mathrm{w})=1$ and $\nu$ is a w-element.

THEOREM 2.9. Suppose that the prime $u$ is a factor of $|G|$ which satisfies the hypotheses of (2.8) plus the extra condition that either $u$ is a q-primitive divisor of $q^{d}-1$ or $u$ is a q-primitive divisor of $q^{t}-1$ where $d=2 t$ and $q$ is even. Let $\mathrm{G}$ be a solvable geometrically primitive subgroup of the linear translation complement. Then no solvable minimal non-f.p.f. group with respect to $G$ is a w-group for a prime w.

PROOF. Suppose that $W$ does exist with the usual properties. By Huppert, Satz 13.7 [5] $\mathrm{W}$ is a central product $\mathrm{W}_{1} \mathrm{~W}_{2} \ldots \mathrm{W}_{\mathrm{k}}$ where $\mathrm{W}_{i} / \mathrm{Z}(\mathrm{W})$ is elementary abelian of order $w^{2}$. If $\sigma \in W$ then $\sigma^{W} \in Z(W)=W_{0}$. Hence if $V(\sigma)$ is nontrivial, then $|\sigma|=w$ since $W_{0}$ must be fixed point free. Furthermore the conjugate of $\sigma$ with respect to an element of $W$ is either $\sigma$ or the product of $\sigma$ with an element of $W_{0}$. It follows that $\sigma$ has exactly $w$ conjugates with respect to $W$ and that $\pi$ as a vector space is the direct sum of $w$ copies of $V(\sigma)$ so that $\operatorname{dim} V(\sigma)=2 d \div w$. If $q$ is even, $w \neq 2$. If $\operatorname{dim} v(\sigma)=e$, then the prime $u$ does not divide $q^{e}-1$ if $u$ is a $q$-primitive divisor of $q^{d}-1$ or if $q^{t}-1$ with $q$ even.

By (2.8) each element of order $u$ in $G$ must leave $V(\sigma)$ invariant; in the present situation such an element must fix $V(\sigma)$ pointwise.

Thus if $W$ exists the normal subgroup generated by the u-elements fixes some nontrivial subspace pointwise and $G$ cannot be geometrically irreducible. Hence W cannot exist if $\mathrm{G}$ is geometrically irreducible and geometrically primitive.

REMARK. A prime q-primitive divisor of $q^{d}-1$ is not ncessarily larger than $d$ or even larger than the largest prime power factor of $d$. However this is "usually" the case at least for small $q$ and $d$. Note that $2^{6}-1$ has no 2-primitive divisors but $2^{3}-1$ has a primitive divisor larger than 6 . 


\section{BOUNDS ON THE DIMENSION.}

It is probably well known from classical representation theory that the dimension of the smallest complex representation of PSL $(2, u)$ is $\frac{1}{2}(u-1)$. For a group acting on a vector space over a finite field with characteristic prime to $u$ this is part of the results of Harris and Hering [2] even if the representation cannot be obtained from a complex representation. This section represents a slight sharpening of this part of the results of Harris and Hering [2] for the case where the group is part of the translation complement of a finite translation plane. Furthermore our methods are relatively elementary. We consider this to be an asset. The group $W$ in this section does not play the same role as the group W of Section 2.

LEMMA 3.1. Let $\mathrm{W}$ be a group of prime order $\mathrm{w}$ acting on a vector space of finite dimension d. Suppose that (1) does not fix pointwise any proper subspace of V. (2) There exists a nonsingular linear transformation $\lambda$ on $V$ which normalizes and induces by conjugation a regular automorphism group of order $h_{0}$ on $W$ (i.e. $\lambda^{i}$ centralizes no nontrivial element of $w$ for $i<0<h_{0}$ but $\lambda^{i}$ centralizes $W$ for $\left.i=h_{0}\right)$.

Then $h_{0}$ divides $d$.

PROOF. We shall show that $\lambda$ permutes the eigenvalues of $\sigma$, where $W=\langle\sigma\rangle$. Let $\mathrm{K}$ be an extension of GF(q) which contains all of the eigenvalues of $\sigma$. We can embed $V$ in a vector space $V^{*}$ of the same dimension as $V$ but where $V^{*}$ is a vector space over $K$. Let $\theta$ be an element of $K$ which is an eigenvalue of $\sigma$. Then the eigenspace (in $V^{*}$ ) belonging to $\theta$ is identical with $V\left(\sigma \theta^{-1}\right)$, the subspace of $\mathrm{V}^{*}$ pointwise fixed by $\sigma \theta^{-1}$. Now $\lambda^{-1} \sigma \lambda=\sigma^{\mathrm{a}}$ for some integer a and $\lambda^{-1}\left(\sigma \theta^{-1}\right) \lambda=\sigma^{a} \theta^{-1}$ so $\mathrm{V}\left(\sigma \theta^{-1}\right) \lambda=\mathrm{V}\left(\sigma^{\mathrm{a}} \theta^{-1}\right)$ if $\sigma^{\mathrm{ab}}=1, \mathrm{~V}\left(\sigma^{\mathrm{a}} \theta^{-1}\right)=\mathrm{V}\left(\sigma \theta^{-\mathrm{b}}\right)$ and is the eigenspace to $\theta^{b}$. The two eigenspaces are disjoint unless they are identical. They are identical only if $\sigma \theta^{-1}=\sigma \theta^{-b}$ so that $\theta=\theta^{b}$. But then $\sigma^{a}=\sigma$ which cannot happen since $\lambda$ induces an automorphism of order $\frac{1}{2}(w-1)$. In a similar fashion, $\lambda$ permutes eigenvalues $\neq 1$ in cycles of length $h_{0}$. Now $v^{*}$ is a direct sum of eigenspaces of $\sigma$ and $\lambda$ permutes these eigenspaces in orbits of length $h_{0}=\frac{1}{2}(w-1)$.

Hence $h_{0}$ divides dim $v$. 
THEOREM 3.2. Let $G$ be a subgroup of the linear translation complement of a translation plane with kernel GF( $q)$, order $q^{d}$. Let $w$ be an odd prime relatively prime to $q$. Suppose that $G$ has a normal subgroup $W$ which is elementary abelian of order $w^{a}$ and that $G$ induces a cyclic automorphism group on $W$ by conjugation so that the nontrivial elements of $\mathrm{W}$ fall into exactly conjugate classes each of length $\frac{1}{2}\left(w^{a}-1\right)$. Then either $w^{a}=4 d+1$ or $w^{a} \leqq 2 d+1$. If $v(w)$ is trivial, then $w^{a}-1$ divides $4 d$.

PROOF. Note that $G$ is acting on a vector space of dimension $2 d$ over $G F(q)$.

Consider the case where $a>1$. There may or may not be some nontrivial subspace $\mathrm{V}(\mathrm{W})$ pointwise fixed by $\mathrm{W}$. If $\mathrm{V}(\mathrm{W})$ is nontrivial, $W$ has some complementary space on which it acts faithfully.

Let $\mathrm{V}$ be a vector space on which $\mathrm{W}$ acts faithfully. (The following is suggested by the proof of Lemma 1.3 in Harris and Hering [2].)

There exists an element $\lambda$ such that $\lambda$ induces a group of automorphisms on $W$ of order $\frac{1}{2}\left(w^{a}-1\right)$. Let $G_{1}=\langle\lambda, w\rangle$ and let $\eta=\left\langle\lambda^{e}, w\right\rangle$, where $e=\frac{1}{2}\left(w^{a}-1\right)$. Since $\lambda^{e}$ centralizes $w, \eta$ is abelian. Then Clifford's theorem implies that $V$ is a direct sum $v_{1} \oplus \ldots \oplus v_{k}$ of homogeneous $\eta$-spaces. If a $>1$ some element $\sigma$ of $w$ is not f.p.f. on $v_{1}$. But $\eta$ is abelian so $\sigma$ fixes a minimal $\eta$-space pointwise and, since $v_{1}$ is a homogeneous $\eta$-space, $v_{1}$ is pointwise fixed by $\sigma$. An f.p.f. elementary abelian w-group must have order $w$ so $W$ must induce a group of order $w$ on $w_{1}--i$.e. $w_{1}$ is pointwise fixed by a group of order $w^{a-1}$. Thus $v_{1}, \ldots, v_{k}$ respectively are pointwise fixed by conjugate subgroups of $\mathrm{W}$ having order $\mathrm{w}^{\mathrm{a}-1}$. The number of subgroups of order $\mathrm{w}^{\mathrm{a}-1}$ in a conjugate class is the same as the number of subgroups of order $w$ in a conjugate class and is either $\left(w^{a}-1\right)(w-1)^{-1}$ or $\frac{1}{2}\left(w^{a}-1\right)(w-1)^{-1}$ depending on whether a subgroup of order whas 2 or 1 conjugate classes of nontrivial elements.

By the previous Lemma $h_{0}=\frac{1}{2}(w-1)$ or $w-1$ divides $\operatorname{dim} v_{1}$. Hence $\mathrm{V}_{0} \oplus \mathrm{V}_{1} \oplus \ldots \oplus \mathrm{V}_{\mathrm{h}}$ is invariant under $\mathrm{G}$ and $\operatorname{dim}\left[\mathrm{v}_{1} \oplus \ldots \oplus \mathrm{V}_{\mathrm{h}}\right]$ is a multiple of $\frac{1}{2}\left(w^{a}-1\right)$. Furthermore if $\pi=V_{0} \oplus \mathrm{V}_{1} \ldots \leftrightarrow \mathrm{V}_{\mathrm{h}} \oplus \mathrm{V}^{\prime}$ then the argument can be repeated to show that $V^{\prime}$ contains a direct sum $V_{1}^{\prime} \ldots \oplus V_{h}^{\prime}$ where $\frac{1}{2}\left(w^{a}-1\right)$ divides $\operatorname{dim}\left[V_{1}^{\prime} \oplus \ldots \oplus V_{h}^{\prime}\right] . \quad A 11$ 
of this holds for $a=1$ again by the previous Lemma. By induction, $\pi$ is the direct sum of $\mathrm{V}_{0}$ and a subspace whose dimension is divisible by $\frac{1}{2}\left(w^{a}-1\right)$. If $v_{0}$ is trivial, then $\frac{1}{2}\left(w^{a}-1\right)$ divides $2 d$ or $4 d \equiv 0 \bmod w^{a}-1$. In this case either $4 d=w^{a}-1$ or $2 d \geqq w^{a}-1$.

Now suppose that $v_{0}$ is not trivial. Here we make our first use of the fact that $G$ is acting on a translation plane. If $\ell$ is any component such that $v_{0} \cap \ell$ is nontrivial, then $\ell$ is invariant under $W$. Furthermore $V_{0}$ is a subplane iff at least three components are invariant under $W$. With the proper basis, $W$ can be represented by matrices of the form $\left(\begin{array}{ll}A & 0 \\ 0 & A\end{array}\right)$ where the points of $\pi$ are ordered pairs $(x, y)$ from a vector space of dimension $d$; the sets of points for which $x=0, y=0$, $y=x$ respectively are three invariant components. In this case if $v_{i}$ intersects an invariant component it intersects all of them. Hence $h=\frac{1}{2}\left(w^{a}-1\right)$ is less than d.

If $W$ has precisely one invariant component we again conclude $h<d$. (This case doesn't really happen) If $W$ has two invariant components and $\langle\lambda\rangle$ leaves both invariant we again conclude $\mathrm{h}<\mathrm{d}$. If $\mathrm{W}$ has two invariant components in the same orbit under $\lambda$ then $v_{0}$ must intersect both of them nontrivially and we are back to the case where $V_{0}$ is a subplane.

COROLLARY 3.3. Let $G$ be a subgroup of the translation complement for a translation plane of order $q^{d}$ with kernel GF(q). Suppose that $G$ has some normal subgroup $H$ such that $G / H \cong \operatorname{PSL}\left(2, u^{a}\right)$ for some odd prime $u,(|H|, u)=1$ and $(u, q)=1$. Then either $u^{a}=4 d+1$ or $u^{a} \leqq 2 d+1$. If $u^{a}-1$ does not divide $4 d$ then a Sylow u-group in $G$ fixes a nontrivial subplane pointwise.

PROOF. The group $G$ of the previous theorem exists. If $u^{a}-1$ does not divide $4 \mathrm{~d}$, the Sylow $\mathrm{u}-\mathrm{group}$ has at least one fixed component. There is more than one fixed component since $(u, q)=1$ and each fixed component must contain fixed points different from 0 if $\frac{1}{2}\left(u^{a}-1\right)$ does not divide $2 d$.

THEOREM 3.4. Let $\pi$ be a translation plane of order $q^{4}$ and with kernel GF(q) where $q$ is even. Suppose that the translation complement $G$ of $\pi$ contains no affine elations. Then the Sylow 2 groups of $G$ have nilpotency class at most 2 . 
PROOF. Let $S$ be a Sylow 2-group. Since the number of components of the spread is odd, $S$ must fix some component $\ell$ and act faithfully on $\ell$. Furthermore each involution in $\mathrm{S}$ is a Baer involution and hence fixes a 2-space on $\ell$ pointwise. Furthermore if $\sigma$ is any nontrivial element, we must have that $V(\sigma) \cap \ell$ has dimension at most 2 .

Let us restrict ourselves to the representation of $\mathrm{S}$ on $\ell$ by $4 \times 4$ matrices. We can choose a basis so that the elements of $\mathrm{S}$ are represented by upper triangular matrices with $l^{\prime}$ 's on the diagonal. Furthermore we may assume that some involution in the center is represented by a matrix of the form

$$
\sigma=\left(\begin{array}{cccc}
1 & c & c_{1} & c_{2} \\
0 & 1 & c_{3} & c_{4} \\
0 & 0 & 1 & 0 \\
0 & 0 & 0 & 1
\end{array}\right)
$$

But if $\sigma$ is an involution we must have $\left[\left(\begin{array}{ll}1 & c \\ 0 & 1\end{array}\right)+\left(\begin{array}{ll}1 & 0 \\ 0 & 1\end{array}\right)\right]\left(\begin{array}{ll}c_{1} & c_{2} \\ c_{3} & c_{4}\end{array}\right)=\left(\begin{array}{ll}0 & 0 \\ 0 & 0\end{array}\right)$.

Hence either $c_{3}=c_{4}=0$ or $c=0$. In the former case the points $(0,1,0,0)(0,0,1,0)$ and $(0,0,0,1)$ are all fixed contrary to the condition that the dimension of $V(\sigma)$ is equal to 2. Hence $c=0$. In abbreviated form $\sigma=\left(\begin{array}{ll}I & C \\ 0 & I\end{array}\right)$ where the capital letters are 2 by 2 matrices. By a further change of basis we can take $\sigma=\left(\begin{array}{ll}I & I \\ 0 & I\end{array}\right)$. Now the condition that $\left(\begin{array}{ll}A & E \\ 0 & I\end{array}\right)$ commute with $\sigma$ is that $A=R$. We may take $A=\left(\begin{array}{ll}1 & a \\ 0 & 1\end{array}\right)$. The reader may verify that the commutator of $\left(\begin{array}{ll}C_{11} & C_{12} \\ 0 & C_{11}\end{array}\right)$ and $\left(\begin{array}{cc}D_{11} & D_{12} \\ 0 & D_{11}\end{array}\right) \quad$ has the form $\left(\begin{array}{cc}I & E \\ 0 & I\end{array}\right)$

where $E=D_{11} D_{12}+D_{11} C_{11} C_{12} D_{11}+C_{11} D_{11} D_{12} C_{11}+C_{11} C_{12} \quad$ (using the fact that $\mathrm{C}_{11}, \mathrm{D}_{11}$ are commuting involutions).

If we set $D_{11}=\left(\begin{array}{ll}1 & d \\ 0 & 1\end{array}\right)$ and $C_{11}=\left(\begin{array}{ll}1 & c \\ 0 & 1\end{array}\right)$ it turns out that the element in the left hand corner of $E$ is 0 . The commutator of $\left(\begin{array}{ll}I & E \\ 0 & I\end{array}\right)$ with respect to a general element then has the form $\left(\begin{array}{ll}I & K \\ 0 & I\end{array}\right)$ where $K$ has the form $\left(\begin{array}{cc}0 & I \\ f_{11} & f_{12} \\ 0 & 0\end{array}\right)$. But then $\left(\begin{array}{ll}I & K \\ 0 & I\end{array}\right)$ fixes the vectors $(0,1,0,0)(0,0,1,0)$ and $(0,0,0,1)$. It follows that $K=0$ and thus the commutator of three elements is the identity. 


\section{APPLICATIONS.}

As before $\mathrm{G}$ is understood to be a subgroup of the linear translation complement of a translation plane with kernel $G F(q)$ and order $q^{d}$. We shall assume that $G$ is geometrically primitive and $G_{0}$ will denote a minimal nonsolvable normal subgroup of $G$ if $G$ is nonsolvable.

As we have pointed out in several previous papers when $q$ and $d$ are both odd and $G$ is nonsolvable $G_{0}$ contains a normal subgroup $H$ of $G$ such that $G_{0} / H$ is $\operatorname{PSL}(2, u)$ for some odd $u$ or $G_{0} / \mathrm{H}$ is $A_{7}$.

Consider the case $q=d=5$. Applying (2.7), (2.3), (2.4) and the remarks preceding (2.7) we can say that $H$ is a Schur multiplier for $G_{0}$. By (4.8) of [10], each odd prime factor of $G$ must divide $5\left(5^{5}+1\right)\left(5^{5}-1\right)\left(5^{4}-1\right)\left(5^{3}-1\right)$ so $A_{7}$ does not apply. Hence $G_{0}=S L(2, u)$ for some odd $u$. If $(u, 5)=1$ then by $(3.2)$ either $u=4.5+1=21$ or $u \leqq 11$. Since 21 is composite we cannot have $u=21$. Thus $u=11,9$, or a power of 5 . If we put in the condition that $G$ has a subgroup fixing some component $\ell$ and transitive on nonzero vectors of $\ell$, then $|G|$ is divisible by the prime factor 71 of $5^{5}-1$. (This condition arises naturally in the investigation of rank three planes.) We leave it to the reader to verify that in this case the only possibility is $\mathrm{u}=5^{2}$ so that the plane is Desarguesian.

A similar argument works for $q=3, d=5$ if we again assume $G$ has a subgroup fixing and transitive on some component $\ell$ of the spread. These are special cases; our results appear to be the most useful in narrowing down the possibilities for groups in particular situations.

Consider the case where $q$ is even and $d=4$. If $G$ contains affine elations (shears) we can apply Hering's results on the groups generated by elations [3], so assume that $G$ has no affine elations. By (3.4) the Sylow 2-groups have nilpotency class at most 2 .

According to Walter [11] the nonsolvable simple groups with abelian Sylow 2-groups are in the following 1ist: $\operatorname{PSL}(2, u), u>3 u \equiv 3$ or $5 \bmod 8$ or $u=2^{s}$; $J(11)$, Ree type $\left(R_{1}(u)\right)$.

According to Gilman and Gorenstein [1], the simple groups with Sylow 2-groups 
of nilpotency class 2 are $\operatorname{PSL}(2, u) u \equiv 7,9 \bmod 16, A_{7}, \operatorname{Sz}\left(2^{n}\right), U_{3}\left(2^{3}\right), \operatorname{PSL}\left(3,2^{n}\right)$, $\operatorname{PSp}\left(4,2^{n}\right) \mathrm{n} \geqq 2$.

We note that $\operatorname{PSL}(3,2)=\operatorname{PSL}(2,7)$ acts on a plane of order 16 but is geometrically reducible [7].

To illustrate the methods of this paper, we restrict ourselves to the cases where $G_{0} / H=P S L(2, u) \quad u \equiv 3$ or $5 \bmod 8$ or $u \equiv 7$ or $9 \bmod 16$ or $G$ is solvable. Note that if $q$ is even and $G$ is geometrically primitive then $G$ can have no normal 2-groups. By (2.6) and (2.7) every solvable normal subgroup will be fixed point free or metacyclic.

A fixed point free group on a vector space over a field of characteristic 2 must have odd order. Except possibly if $u=9$ the Schur multiplier for $\operatorname{PSL}(2, u)$ ( $u$ odd) has order 2. In the present context we actually have that $H$ of (2.3) must be trivial so that $G_{0}=\operatorname{PSL}(2, u)$. By (3.2) $u=4 \cdot 4+1=17$ or $\mathrm{u} \leqq 4 \cdot 2+1=9$. But $17 \equiv 1 \bmod 16$ so $u \leqq 9$. Since $\operatorname{PSL}(2,5)=\operatorname{PSL}(2,4)$ the only cases left are $\operatorname{PSL}(2,9)$ and $\operatorname{PSL}(2,7)$.

\section{REFERENCES}

1. GILMAN, R. and GORENSTEIN, D. Finite Groups with Sylow 2-Groups of Class Two, I, II, Trans. Am. Math. Soc. 207 (1975), 1-101, 103-125.

2. HARRIS, M. and HERING, C., On the Smallest Degrees of Projective Representations of the Groups PSL $(n, q)$, Can. J. Math. 23 (1971), 90-102.

3. HERING, C. On Shears of Translation Planes, Abh. Math. Sem. Hamb. 37 (1972), 258-268.

4. HUPPERT, B. Lineare Auflosbare Gruppen Math, Math. Z. 67 (1957), 479-518.

5. HUPPERT, B. Endliche Gruppen I, Springer-Verlag, Berlin and New York (1967).

6. KALLAHER, M. and OSTROM, T.G. Collineation Groups Irreducible on the Components of a Translation Plane, Geom. Ded. 9 (1980), 153-194.

7. LORIMER, P. A Projective Plane of Order 16, J. Comb. Thy - (A) 16 (1974), 334-347.

8. OSTROM, T.G. Solvable Linear Groups on Vector Spaces whose Dimension is the Product of Two Primes, Aeq. Math. 18 (1978), 77-102.

9. Ostrom, T.G. Translation Planes of Odd Order and Odd Dimension, Int. J. Math. and Math Sciences $\underline{2}$ (1979), 187-208.

10. Translation Planes of Even Order in which the Dimension has only One Udd Factor, Int. J. of Math and Math. Sciences.

11. WALTER, J. The Characterization of Finite Groups with Abelian Sylow 2-Groups, Ann. Math. 89 (1969), 405-514. 


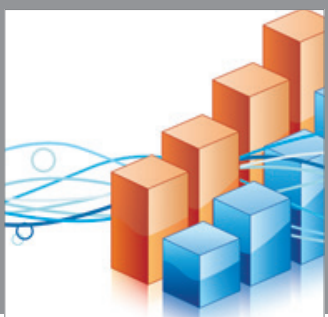

Advances in

Operations Research

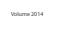

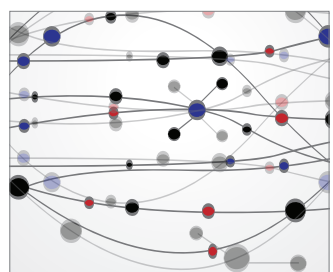

\section{The Scientific} World Journal
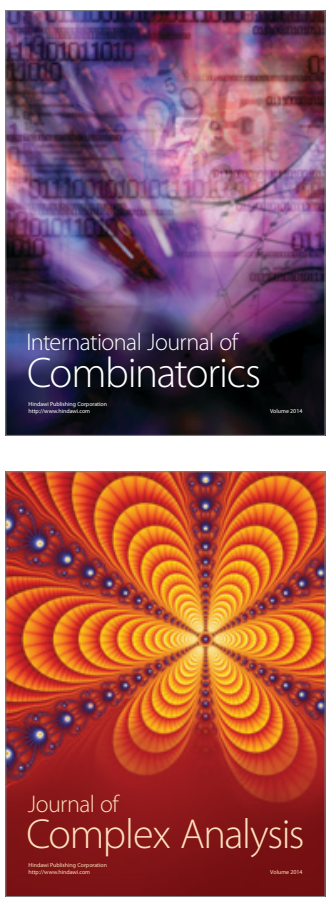

International Journal of

Mathematics and

Mathematical

Sciences
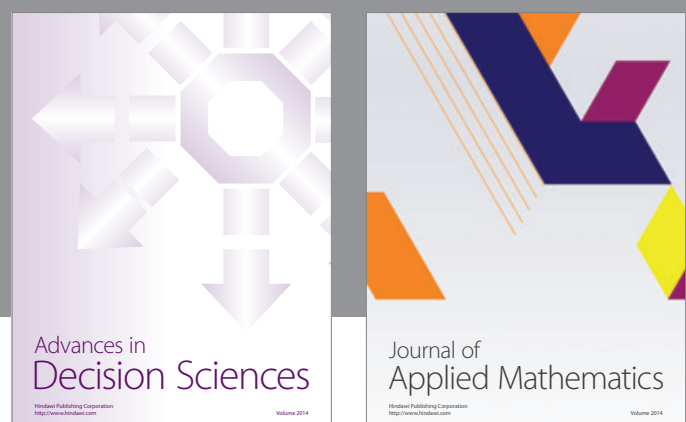

Journal of

Applied Mathematics
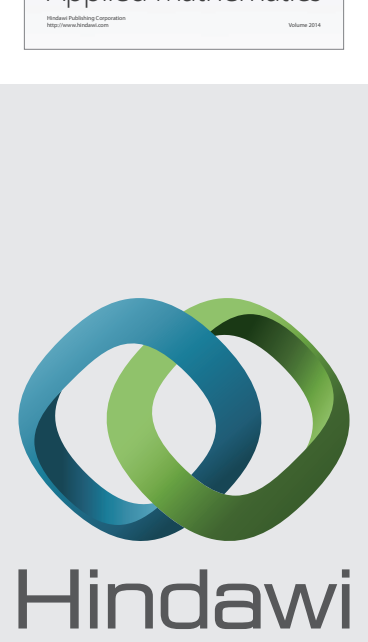

Submit your manuscripts at http://www.hindawi.com
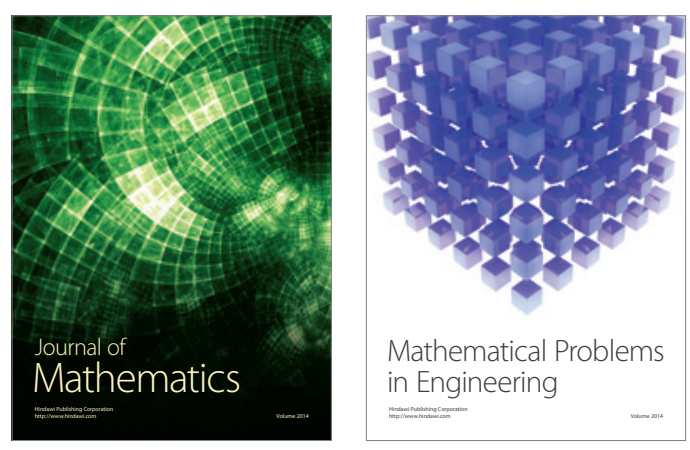

Mathematical Problems in Engineering
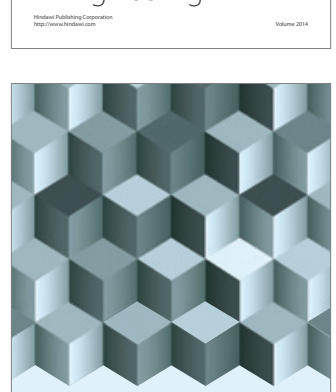

Journal of

Function Spaces
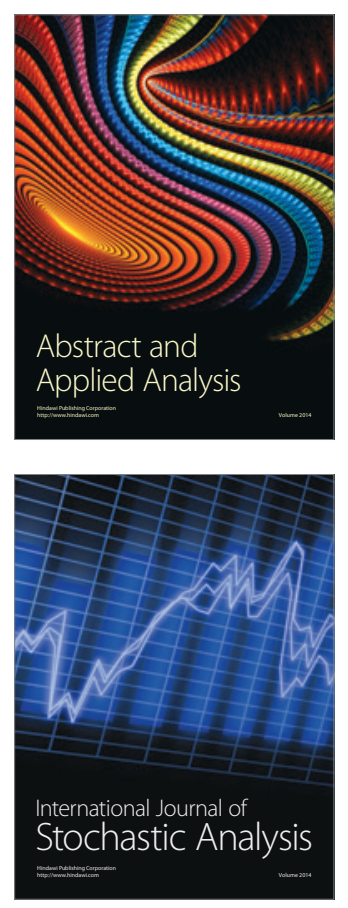

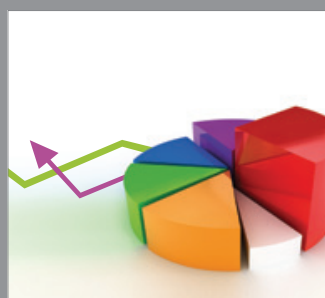

ournal of

Probability and Statistics

Promensencen
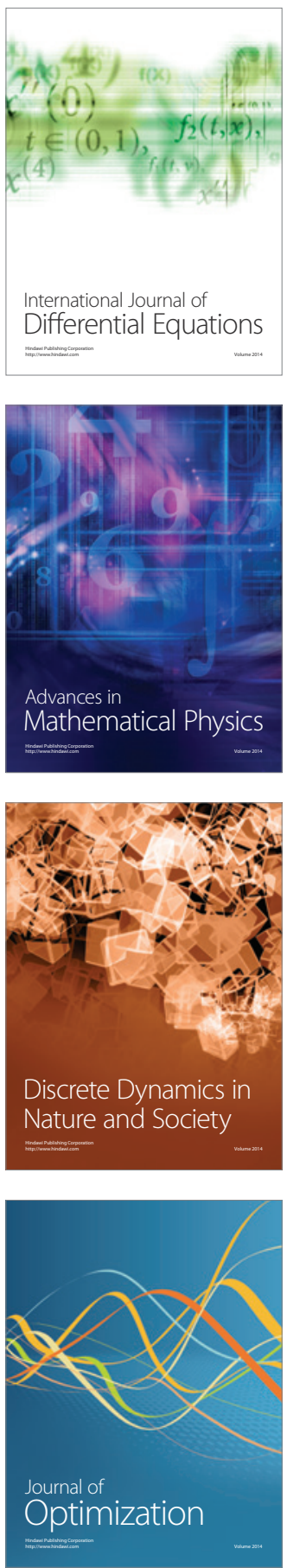